\title{
Wideband-tuneable, nanotube mode-locked, fibre laser
}

\author{
F. WANG ${ }^{1}$, A. G. ROZHIN ${ }^{1}$, V. SCARDACl ${ }^{1}$, Z. SUN ${ }^{1}$, F. HENNRICH², I. H. WHITE ${ }^{1}$, W. I. MILNE \\ AND A. C. FERRARI ${ }^{\text {* }}$
}

${ }^{1}$ Department of Engineering, University of Cambridge, 9 JJ Thomson Avenue, Cambridge, CB3 OFA, UK

${ }^{2}$ Institut für Nanotechnologie Forschungszentrum Karlsruhe, 76021 Karlsruhe, Germany

*e-mail: acf26@eng.cam.ac.uk

Published online: 2 November 2008; doi:10.1038/nnano.2008.312

Ultrashort-pulse lasers with spectral tuning capability have widespread applications in fields such as spectroscopy, biomedical research and telecommunications ${ }^{1-3}$. Mode-locked fibre lasers are convenient and powerful sources of ultrashort pulses $^{4}$, and the inclusion of a broadband saturable absorber as a passive optical switch inside the laser cavity may offer tuneability over a range of wavelengths ${ }^{5}$. Semiconductor saturable absorber mirrors are widely used in fibre lasers ${ }^{4-6}$, but their operating range is typically limited to a few tens of nanometres ${ }^{7,8}$, and their fabrication can be challenging in the 1.3-1.5 $\mu \mathrm{m}$ wavelength region used for optical communications ${ }^{9,10}$. Single-walled carbon nanotubes are excellent saturable absorbers because of their subpicosecond recovery time, low saturation intensity, polarization insensitivity, and mechanical and environmental robustness ${ }^{11-16}$. Here, we engineer a nanotube-polycarbonate film with a wide bandwidth $(>300 \mathrm{~nm})$ around $1.55 \mu \mathrm{m}$, and then use it to demonstrate a 2.4 ps $\mathrm{Er}^{3+}$-doped fibre laser that is tuneable from 1,518 to $1,558 \mathrm{~nm}$. In principle, different diameters and chiralities of nanotubes could be combined to enable compact, mode-locked fibre lasers that are tuneable over a much broader range of wavelengths than other systems.

The development of compact, diode-pumped, ultrafast fibre lasers as alternatives for bulk solid-state lasers is fast progressing. To date, short pulse generation has been particularly effective using passive mode-locking techniques ${ }^{4}$. At present, the dominant technology in passively mode-locked fibre lasers is based on semiconductor saturable absorber mirrors (SESAMs) ${ }^{6}$. Conventional SESAMs use III-V semiconductor multiple quantum wells grown on distributed Bragg reflectors (DBRs) ${ }^{3}$. Their fabrication involves molecular beam epitaxy $(\mathrm{MBE})^{6}$. To reduce the relaxation time to sub-picosecond levels, either postgrowth ion-implantation or low-temperature growth is normally required $^{6,17}$. Furthermore, SESAMs are based on a resonant nonlinearity, which tends to limit wavelength tuneability ${ }^{18,19}$ for the shortest pulse lasers ${ }^{20}$. Their operating bandwidth is further limited by the bottom DBR section, which has a finite bandwidth for high reflectivity ${ }^{19}$. For example, the bandwidth of conventional $\mathrm{Al}_{x} \mathrm{Ga}_{1-x} \mathrm{As} / \mathrm{AlAs}$ SESAMs is limited to about $60 \mathrm{~nm}$ by the bottom Bragg mirrors ${ }^{21}$. Wider bandwidth, $\gtrsim 200 \mathrm{~nm}$, was achieved using novel material pairs with larger refractive index difference (for example, $\left.\mathrm{AlGaAs} / \mathrm{CaF}_{2}\right)^{21}$, or by replacing the DBRs with metallic mirrors ${ }^{22}$. However, so far, no widely tuneable mode-locked laser has been reported using these novel structures. Trade-offs between design parameters have to be made in order to obtain targeted device characteristics ${ }^{10}$. A tuning range over $100 \mathrm{~nm}$ was achieved by SESAMs in solidstate and fibre lasers ${ }^{18,23}$. The widest was $125 \mathrm{~nm}$, for a Yb-doped fibre laser operating at $1 \mu \mathrm{m}$. However, two SESAMs with complementary spectral properties had to be used ${ }^{23}$. Thus, much simplified and cost-effective saturable absorbers with wideband tuneability are needed.

Single-walled carbon nanotubes (SWNTs) are direct-bandgap materials, with a gap that depends on diameter and chirality ${ }^{24}$ Many of the applications that might make use of the novel electronic properties of these materials require individual nanotubes with a given chirality for optimum performance, but it is still not possible to grow nanotubes with well-defined chiralities. However, in optoelectronic applications, this disadvantage can be turned into an advantage. SWNTs show large optical nonlinearity ${ }^{25}$, ultrafast carrier relaxation time $^{26}$ and high damage threshold ${ }^{27}$. They are compatible with optical fibres, and mode-locked laser operation at $\sim 1.5 \mu \mathrm{m}$ has been demonstrated ${ }^{15,16,27-32}$. The variation of nonlinear absorption is determined by the number of tubes in resonance with the incident light. However, even for a wavelength detuning of up to $\sim 200 \mathrm{~nm}$ from the peak resonance, appreciable saturable absorption can still be observed ${ }^{25}$. This implies great potential for wideband tuneable lasers. Previous mode-lockers relied on SWNT solutions ${ }^{13}$, layers grown or spray-coated over optical parts $^{29}$, or polymer composites ${ }^{15,28}$. The last of these, polymer composites, allow homogeneous dispersion and easy integration.

To allow low-cost mounting and ease of use, we fabricated a $\sim 40$ - $\mu$ m-thick SWNT-polycarbonate composite with a broad $(\sim 300 \mathrm{~nm})$ absorption band in the $1.5 \mu \mathrm{m}$ spectral region (described in the Methods and shown in Fig. 1a). This was achieved by using SWNTs with diameters ranging from 1 to $1.3 \mathrm{~nm}$. Note that pure polycarbonate has a low absorption around 1,550 nm (Fig. 1a).

We first investigated the wavelength-dependent saturable absorption within the $\mathrm{Er}^{3+}$-doped fibre gain bandwidth (see Methods). Figure $1 \mathrm{~b}$ plots the measured loss as a function of average pump power at different pump wavelengths. All loss curves show a saturation depth of about $0.3 \mathrm{~dB}$, against a linear loss of $\sim 2.6 \mathrm{~dB}$ (or, equivalently, a $4 \%$ increase in transmittance from a small-signal transmission of 55\%). A slight decrease of modulation depth is detected towards shorter wavelengths, possibly caused by the mismatch between the pump wavelengths 

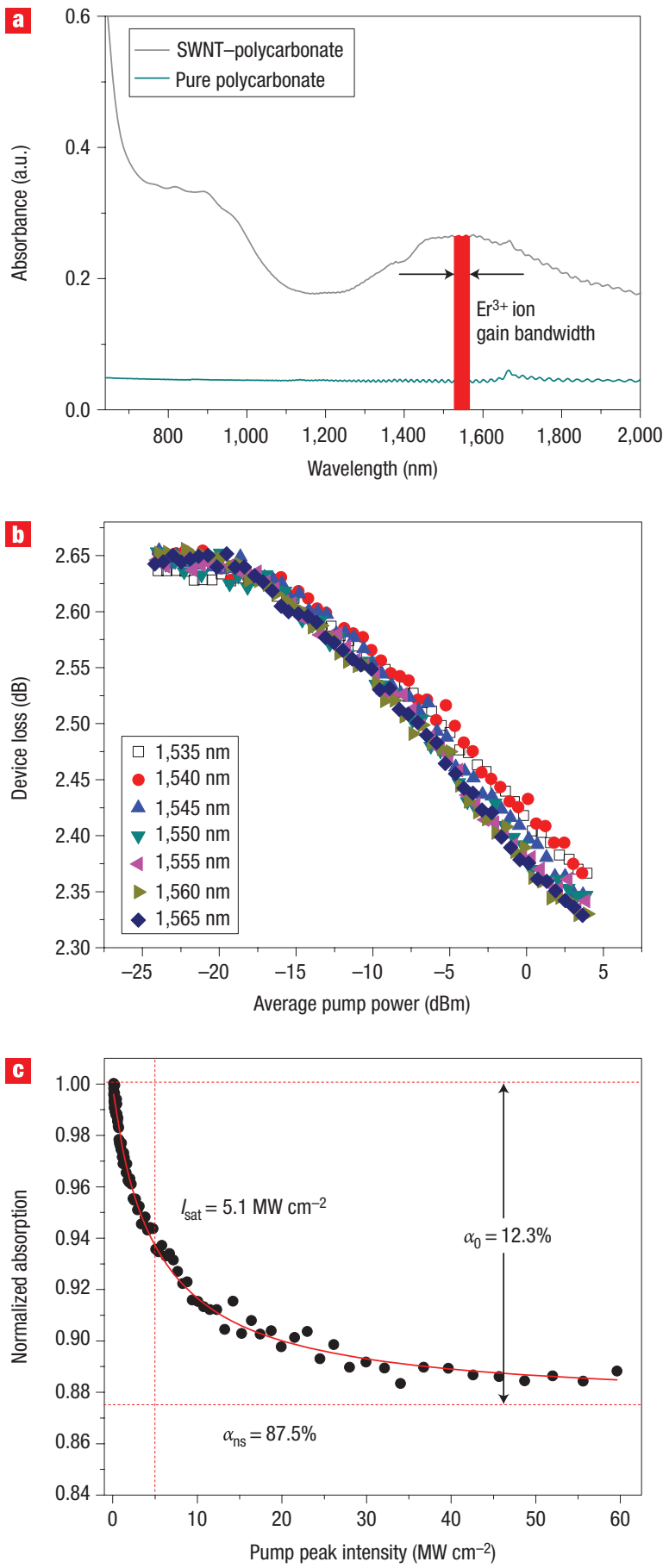

Figure 1 Optical characterization of the composite films. a, Absorption spectrum of the SWNT-polycarbonate composite and pure polycarbonate. The red stripe shows the spectral gain region of the $\mathrm{Er}^{3+}$-doped fibre. $\mathbf{b}$, Nonlinear absorption measurements of our SWNT saturable absorber at seven different wavelengths. The pump power ranges from $3 \mu \mathrm{W}$ to $\sim 3 \mathrm{~mW}$. c, Typical normalized absorption of the SWNT saturable absorber as a function of pump pulse peak intensity. Data correspond to the pump at 1,550 nm (red line: fitted curve according to equation (1)).

and the peak absorption of our SWNTs $(\sim 1,550 \mathrm{~nm})$. Further wavelength detuning would lead to a more pronounced decrease in modulation depth. The data for normalized absorption

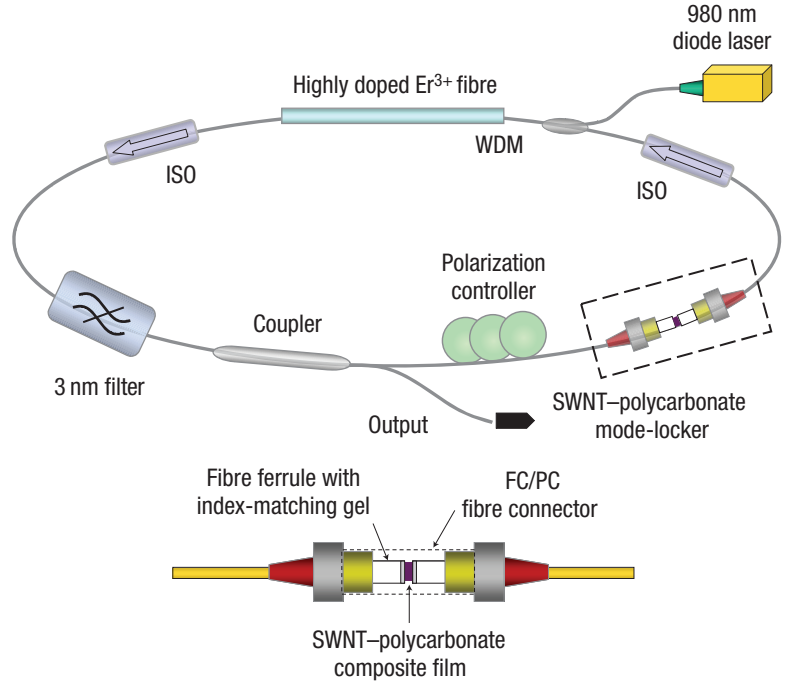

Figure 2 Laser setup and mode-locker assembly. This schematic shows the standard fibre-optic components such as an optical isolator (ISO), a wavelength division multiplexer (WDM), a power splitter and a polarization controller that are found in the ring cavity. The total length of the cavity is about $13.3 \mathrm{~m}$. The basic operation of the laser is discussed in the Methods.

are then fitted according to a simple two-level saturable absorber model ${ }^{33}$ :

$$
\alpha(I)=\frac{\alpha_{0}}{1+I / I_{\mathrm{sat}}}+\alpha_{\mathrm{ns}}
$$

where $\alpha(I)$ is the intensity-dependent absorption coefficient, and $\alpha_{0}, \alpha_{\text {ns }}$ and $I_{\text {sat }}$ are the linear limit of saturable absorption, nonsaturable absorption and saturation intensity, respectively. This gives an average saturable absorption (modulation depth) of $\sim 12 \%$ and an average saturation intensity of $\sim 5.8 \mathrm{MW} \mathrm{cm}^{-2}$ in the spectral range investigated. As an example, a typical nonlinear absorption curve for $1,550 \mathrm{~nm}$ excitation is shown in Fig. 1c. At this specific wavelength, $\alpha_{0}$ and $I_{\text {sat }}$ are $12.3 \%$ and $5.1 \mathrm{MW} \mathrm{cm}^{-2}$, respectively. The modulation depth and saturation intensity for our devices are comparable with those of SESAMs ${ }^{6}$, but the nonsaturable loss is larger. This is tolerable for fibre lasers with a relatively large single round-trip gain coefficient ${ }^{27}$. Yet, it degrades the mode-locking performance, resulting in less output power or longer pulses. In our case, the contributions to the non-saturable losses may include residual absorption due to amorphous carbon, metal catalysts and tubes not in resonance with the incident light, scattering from residual bundles, absorption and refraction from the polymer matrix ${ }^{16}$, and linear coupling loss between fibre ends $^{34}$. By further engineering film thickness and nanotube loadings and alignment, devices with minimized non-saturable loss could be obtained.

Figure 2 shows the setup of the tuneable laser and the schematic of the mode-locker device. Wavelength tuning is provided by the intracavity filter. By changing the tilt angle it is possible to tune the filter pass-band from 1,535 to $1,565 \mathrm{~nm}$. Moreover, we find that the filter can also work from 1,515 to $1,535 \mathrm{~nm}$, but at the expense of increased insertion losses. The $\mathrm{Er}^{3+}$-doped fibre is typically used to amplify light in the 1,530-1,560 nm wavelength range. However, we can extend the continuous-wave lasing wavelength down to $1,518 \mathrm{~nm}$ by feeding more pump power to 

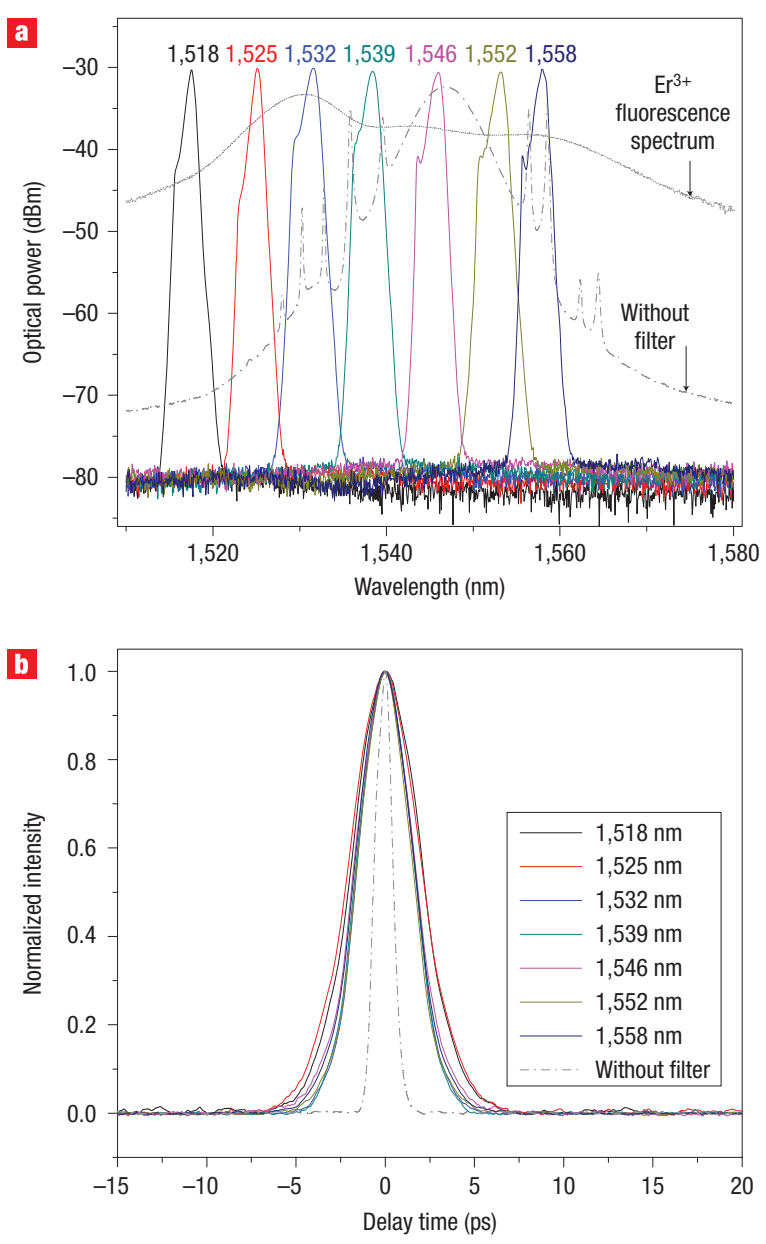

Figure 3 Wavelength tuning. a, Output spectra at seven different wavelengths. (Dashed line, optical spectrum without bandpass filter; dotted line;

$\mathrm{Er}^{3+}$ fluorescence spectrum). The use of the bandpass filter means that the output spectra have a high signal-to-noise ratio of $\sim 50 \mathrm{~dB}\left(\times 10^{5}\right.$ contrast). No sideband structure is observed due to the spectral limiting effect of the filter ${ }^{35}$. On the other hand, the filter also limits the mode-locking spectral width to an average value of $1.2 \mathrm{~nm}$, which keeps the pulse duration above $1 \mathrm{ps}$. b, Autocorrelation traces of laser output at different central wavelengths. (Dashed line, laser output without bandpass filter.) All traces do not show pedestals (low-intensity backgrounds), indicative of single pulse operation and reflection-free design 5 .

the $\mathrm{Er}^{3+}$-doped fibre. Thus, we study the mode-locking of the current laser over the extended wavelength range 1,518-1,565 nm in order to achieve the maximum possible tuning range. We find that a tuning range of $40 \mathrm{~nm}$, from 1,518 to $1,558 \mathrm{~nm}$, is possible with the current setup. Figure 3a,b illustrates the output spectra and autocorrelation traces at several wavelengths within the tuning range. Our cavity uses an anomalous dispersion fibre, with an overall negative group velocity dispersion, in order to facilitate soliton-like pulse shaping through the interplay of group velocity dispersion and self-phase modulation ${ }^{5}$. In this case, the output pulses are expected to have a sech squared lineshape ${ }^{5}$. Indeed, the data in Fig. $3 b$ are well fitted by a sech squared relation, giving an average pulse duration of 2.39 ps. As can be inferred from Fig. 3a, the laser output power stays relatively flat from 1,518 to $1,558 \mathrm{~nm}$. An average value of $0.36 \mathrm{~mW}$, with a standard deviation of $0.055 \mathrm{~mW}( \pm 15 \%)$ is obtained for the wavelengths within the tuning range. As we use a 50/50 coupler, the incident power onto the mode-locker is the same as the output power. From the average output power and repetition rate, the energy per pulse is $24 \mathrm{pJ}$. Considering an average pulse duration of $2.39 \mathrm{ps}$ and a mode diameter of $10 \mu \mathrm{m}$, we obtain a pulse intensity of $\sim 11.2 \mathrm{MW} \mathrm{cm}^{-2}$, about twice the saturation intensity of our absorber. By comparison, the laser output when no filter is present (at a pump level of $35 \mathrm{~mW}$ ) is also shown in Fig. 3a,b as a dashed line. In this case, the laser mode-locks at $1,545 \mathrm{~nm}$ (the effective gain maximum of the cavity) with sidebands arising from periodic cavity perturbations ${ }^{5}$. The pulse width is $706 \mathrm{fs}$, shortened due to the elimination of intracavity spectral limiting effects.

The current laser does not mode-lock at wavelengths beyond $1,558 \mathrm{~nm}$, but it does at shorter wavelengths outside the filter nominal operating spectral range (such as 1,518 and 1,525 nm). To verify this, we use the amplified spontaneous emission (ASE) from an $\mathrm{Er}^{3+}$-doped fibre amplifier as a wideband optical source to measure the filter pass-band characteristics. We find, as expected, that the longer wavelength pass-band exhibits much worse ripple effects than the shorter wavelength one, resulting in strong narrowband limiting effects. We believe this prevents modelocking at longer wavelengths in the current setup. Combined with the data in Fig. 1a,b, we conclude that the nonlinear absorption of our mode-locker is not the limiting factor. In principle our SWNT saturable absorber could also be used to mode-lock and enable tuning for other telecommunication bands such as the $\mathrm{S}(1,460-1,530 \mathrm{~nm})$ and $\mathrm{L}(1,565-1,624 \mathrm{~nm})$ bands, if gain fibres and tuneable filters at these wavelength ranges are available.

Figure 4 summarizes some of the laser output trends and characteristics within the tuning range. An average pulse duration of 2.39 ps is obtained for different wavelengths (Fig. 4a). Figure 4a also plots the product of the pulse temporal and spectral widths. Different applications demand the shortest pulse duration at a given spectral width. Figure $4 \mathrm{a}$ shows that our typical timebandwidth products range from 0.32 to 0.36 , reasonably close to 0.315 , which corresponds to the shortest pulse duration for a given spectral width, for transform-limited sech squared pulses 5 . Figure $4 \mathrm{~b}$ is the measured output pulse train, with a fundamental repetition rate of $15 \mathrm{MHz}$ (period $\tau=66.7 \mathrm{~ns}$ ). Figure 4c shows the radio frequency (RF) power spectrum of the laser output after optical-to-electrical conversion using a fast photodiode. A $\sim 70 \mathrm{~dB}$ peak-to-background ratio $\left(10^{7}\right.$ contrast) is observed for the fundamental peak at a measurement span of $1 \mathrm{kHz}$ and resolution bandwidth of $10 \mathrm{~Hz}$. This indicates good stability of the mode-locking regime. In Figure $4 \mathrm{~d}$ the wideband RF spectrum up to $1 \mathrm{GHz}$ is shown. The absence of spectral modulation indicates that the tuneable laser is operating in the pure continuous-wave mode-locking regime, where the output pulse train is not subject to low-repetition-rate modulation arising from relaxation oscillations ${ }^{6}$.

In conclusion, our work makes use of SWNTs with a range of diameters and chiralities, harnessing the resulting wideband absorption to produce a wideband-tuneable fibre laser, and turning a major disadvantage of SWNTs for applications in nanoelectronics into an advantage. SWNT-polymer composites are a cost-effective and viable alternative to saturable absorbers based on quantum wells and could form the basis of functional building blocks in future nanoscale photonic integrated circuits.

\section{METHODS}

\section{SWNTS COMPOSITE}

The SWNT-polycarbonate composite was prepared first by dispersing SWNT powders in dichlorobenzene (DCB) in the presence of poly(3-hexylthiophene- 

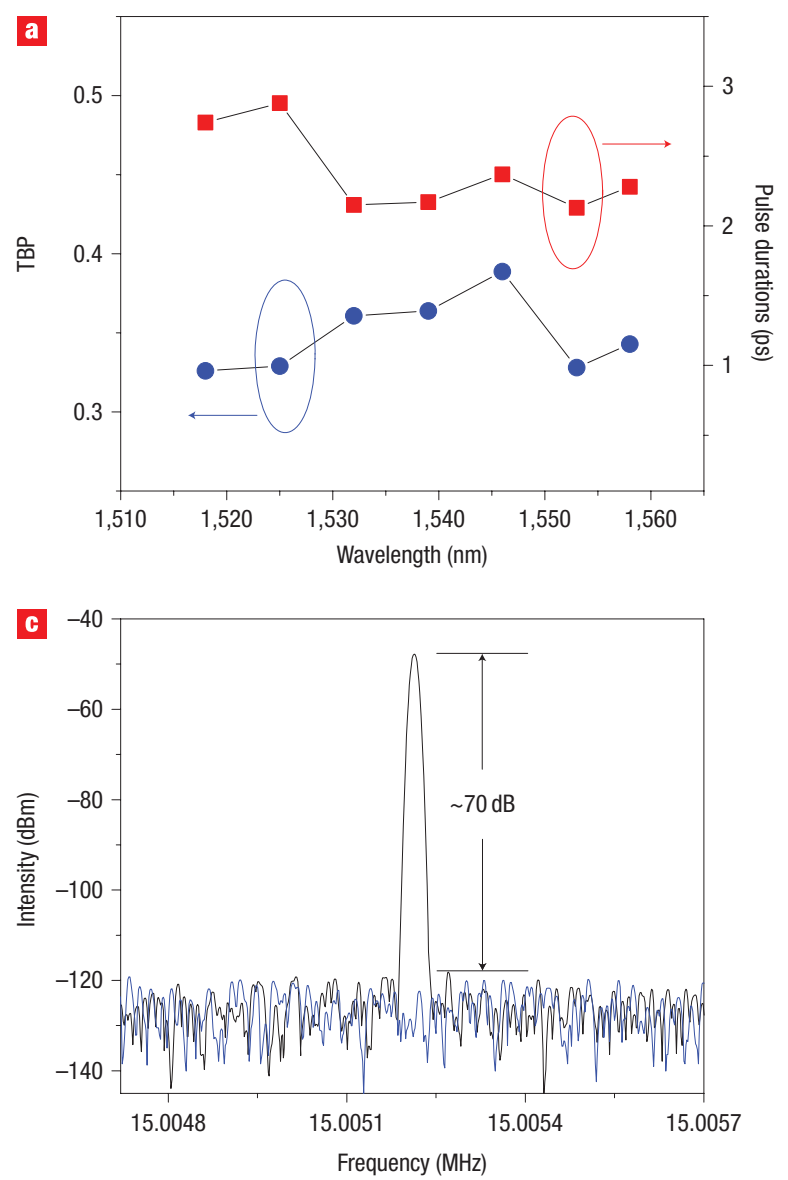
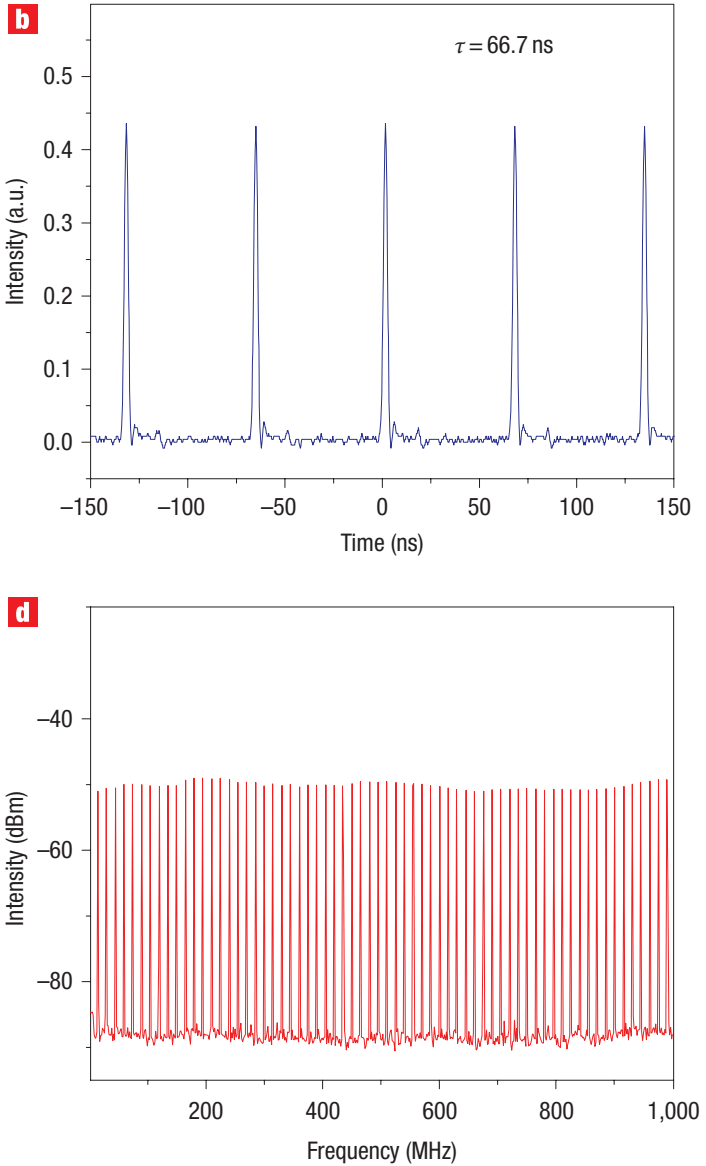

Figure 4 Mode-locking characteristics. a, Output pulse duration and time-bandwidth product (TBP) at different central wavelengths. b, 0scilloscope trace of the typical laser output. c, Fundamental of a typical radio-frequency (RF) spectrum of the laser output after optical-to-electrical conversion. The blue trace depicts the background when the laser is switched off. $\mathbf{d}$, Wideband RF spectrum up to $1 \mathrm{GHz}$.

2,5-diyl) (P3HT) by ultrasonication (Diagenode SA). The solution was then filtered through a $1-\mu \mathrm{m}$ retention filter (Whatman) and centrifuged (Beckman Coulter Optima MaxE Ultracentrifuge, MLA130 fixed-angle rotor) to remove bundles and impurities. Pellets of polycarbonate were dissolved in the solution by ultrasonication. The final mixture was dried at room temperature to form a film with a typical thickness of $40 \mu \mathrm{m}$. No SWNT bundles could be detected under the scrutiny of an optical microscope, thus ensuring minimized scattering losses.

\section{POWER-DEPENDENT ABSORPTION MEASUREMENTS}

Power-dependent absorption measurements were carried out as follows. The SWNT mode-locker assembly was coupled to a $650 \mathrm{fs}$ optical pulse source, tuneable from 1,535 to $1,565 \mathrm{~nm}$. This was achieved by filtering a commercial femtosecond fibre laser (200 fs, $76.9 \mathrm{MHz}$, TOPTICA) using a 3-nm band-pass filter. The laser beam was amplified by an erbium-doped fibre amplifier and a $10 \%$ tap was used to monitor the input power to the mode-locker assembly, containing our composite, and two appropriately calibrated powerheads were programmed to read the input/output power simultaneously.

\section{TUNEABLE LASER EXPERIMENTAL SETUP}

The mode-locker was assembled by sandwiching the free-standing SWNT composite between two fibre ferrules inside a physical contact ferrule connector. The estimated input mode diameter on the composite was $10 \mu \mathrm{m}$. The laser setup is schematically shown in Fig. 2. A 1-m highly doped $\mathrm{Er}^{3+}$ fibre was used as the gain medium. It was pumped by a $980 \mathrm{~nm}$ diode laser using a wavelength division multiplexer. Two isolators were placed at both ends of the amplifier section to maintain unidirectional laser operation. A tuneable filter with a $3 \mathrm{~nm}$ passband was placed after the isolator at the output of the amplification section. Light was then extracted from the cavity using a 50/50 coupler. A polarization controller was used to optimize the mode-locking conditions and the SWNT film placed between this and the isolator, at the input of the amplification section. The total length $L$ of the cavity was $\sim 13.3 \mathrm{~m}$. We could thus estimate the expected repetition rate to be $\sim 15 \mathrm{MHz}$, from $f_{\mathrm{r}}=c /(n L)$, where $f_{\mathrm{r}}$ is the repetition rate, $c$ the velocity of light in vacuum, and $n$ the average refractive index of the cavity $(n \approx 1.5)$.

With the filter in the cavity, the threshold pump power for continuous wave lasing was $\sim 15 \mathrm{~mW}$ at $1,550 \mathrm{~nm}$. When the pump power was increased to $\sim 45 \mathrm{~mW}$, stable mode-locking could be initiated by introducing a disturbance to the polarization controller. Once a stable output was achieved, no further polarization controller adjustment was needed and we could decrease the pump power to $\sim 35 \mathrm{~mW}$ while maintaining mode-locking. For optimal polarization controller settings, the laser self-started with excellent repeatability. The measured repetition rate was $15.01 \mathrm{MHz}$, in agreement with the design parameters.

Received 19 May 2008; accepted 24 September 2008;

published 2 November 2008.

References

1. Letokhov, V. S. Laser biology and medicine. Nature 316, 325-330 (1985).

2. Shah, J. Ultrafast Spectroscopy of Semiconductors and Semiconductor Nanostructures 2nd edn (Springer-Verlag, 1999).

3. Keller, U. Recent developements in compact ultrafast lasers. Nature 424, 831-838 (2003).

4. Digonnet, M. J. F. Rare-Earth-Doped Fiber Lasers and Amplifiers (CRC Press, 2001).

5. Agrawal, G. P. Applications of Nonlinear Fiber Optics (Academic Press, San Diego, 2001).

6. Keller, U. et al. Semiconductor saturable absorber mirrors (SESAMs) for femtosecond to nanosecond pulse generation in solid-state lasers. IEEE J. Sel. Top. Quant. Electron. 2, 435-453 (1996).

7. Grange, R. et al. Nonlinear absorption edge properties of 1.3- $\mu \mathrm{m}$ GaInNAs saturable absorbers. Appl. Phys. Lett. 87, 132103 (2005).

8. Rutz, A. et al. Parameter tunable GaInNAs saturable absorbers for mode locking of solid-state lasers. J. Crys. Grow. 301-302, 570-574 (2007).

9. Calvez, S. et al. GaInNAs/GaAs Bragg-mirror-based structures for novel $1.3 \mu \mathrm{m}$ device applications. J. Crys. Grow. 268, 457-465 (2004). 
10. Rutz, A. et al. $1.5 \mu \mathrm{m}$ GaInNAs semiconductor saturable absorber for passively modelocked solid-state lasers. Electron. Lett. 41, 321-323 (2005)

11. Set, S. Y., Yaguchi, H., Tanaka, Y. \& Jablonski, M. Ultrafast fiber pulsed lasers incorporating carbon nanotubes. IEEE J. Sel. Top. Quant. Electron. 10, 137-146 (2004).

12. Nicholson, J. W., Windeler, R. S. \& DiGiovanni, D. J. Optically driven deposition of single-walled carbon-nanotube saturable absorbers on optical fiber end-faces. Opt. Express 15, 9176-9183 (2007)

13. Il'ichev, N. N., Obraztsova, E. D., Pashinin, P. P., Konov, V. I. \& Garnov, S. V. Self-mode locking in $F_{2}^{-}:$LiF laser by means of a passive switch based on single-wall carbon nanotubes. Quant. Electron. 34, 785-786 (2004)

14. Song, Y. W., Set, S. Y., Yamashita, S., Chee, S. G. \& Kotake, T. 1300-nm pulsed fiber lasers modelocked by purified carbon nanotubes. IEEE Photon. Tech. Lett. 17, 1623-1625 (2005).

15. Dalle Valle, G. et al. Passive mode locking by carbon nanotubes in a femtosecond laser written waveguide laser. Appl. Phys. Lett. 89, 231115 (2006).

16. Rozhin, A. G. et al. Generation of ultra-fast laser pulses using nanotube mode-lockers. Phys. Stat. Sol. (b) 243, 3551-3555 (2006).

17. Haiml, M. et al. Optical nonlinearity in low-temperature-grown GaAs: Microscopic limitations and optimization strategies. Appl. Phys. Lett. 74, 3134-3136 (1999).

18. Jacobovitz-Veselka, G. R., Keller, U. \& Asom, M. T. Broadband fast semiconductor saturable absorber. Opt. Lett. 17, 1791-1793 (1992).

19. Kopf, D., Prasad, A., Zhang, G., Moser, M. \& Keller, U. Broadly tunable femtosecond Cr:LiSAF laser. Opt. Lett. 22, 621-623 (1997).

20. Kärtner, F. X., Jung, I. D. \& Keller, U. Soliton mode-locking with saturable absorbers. IEEE J. Sel. Top. Quant. Electron. 2, 540-556 (1996).

21. Schön, S., Haiml, M. \& Keller, U. Ultrabroadband AIGaAs/CaF semiconductor saturable absorber mirrors. Appl. Phys. Lett. 77, 782-784 (2000).

22. Fluck, R. et al. Broadband saturable absorber for 10 -fs pulse generation. Opt. Lett. 21 , $743-745$ (1996).

23. Gomes, L. A., Orsila, L., Jouhti, T. \& Okhotnikov, O. G. Picosecond SESAM-based ytterbium modelocked fiber lasers. IEEE J. Sel. Top. Quant. Electron. 10, 129-136 (2004).

24. Kataura, H. et al. Optical properties of single-wall carbon nanotubes. Synth. Met. 103, 2555-2558 (1999).
25. Sakakibara, Y., Tatsuura, S., Kataura, H., Tokumoto, M. \& Achiba, Y. Near-infrared saturable absorption of single-wall carbon nanotubes prepared by laser ablation. Jpn J. Appl. Phys. 42, L494-L496 (2003).

26. Lauret, J. S. et al. Ultrafast carrier dynamics in single-wall carbon nanotubes. Phys. Rev. Lett. 90, 057404 (2003).

27. Fong, K. H. et al. Solid-state Er:Yb:glass laser mode-locked by using single-wall carbon nanotube thin film. Opt. Lett. 32, 38-40 (2007).

28. Rozhin, A. G. et al. Sub-200-fs pulsed erbium-doped fiber laser using a carbon nanotubepolyvinylalcohol mode locker. Appl. Phys. Lett. 88, 051118 (2006).

29. Song, Y. W., Yamashita, S., Goh, C. S. \& Set, S. Y. Passively mode-locked lasers with 17.2-GHz fundamental-mode repetition rate pulsed by carbon nanotubes. Opt. Lett. 32, 430-432 (2007).

30. Schibli, T. et al. Ultrashort pulse-generation by saturable absorber mirrors based on polymerembedded carbon nanotubes. Opt. Express 13, 8025-8031 (2005).

31. Song, Y. W., Yamashita, S. \& Maruyama, S. Single-walled carbon nanotubes for high-energy optical pulse formation. Appl. Phys. Lett. 92, 021115 (2008).

32. Kieu, K. \& Mansuripur, M. Femtosecond laser pulse generation with a fiber taper embedded in carbon nanotube/polymer composite. Opt. Lett. 32, 2242-2244 (2007).

33. Garmire, E. Resonant optical nonlinearities in semiconductors. IEEE J. Sel. Top. Quant. Electron. 6 , $1094-1110(2000)$

34. Snyder, A. W. \& Love, J. D. Optical Waveguide Theory (Springer, 1983).

35. Tamura, K., Doerr, C. R., Haus, H. A. \& Ippen, E. P. Soliton fiber ring laser stabilization and tuning with a broad intracavity filter. IEEE Photon. Technol. Lett. 6, 697-699 (1994).

\section{Acknowledgements}

We acknowledge funding from the Isaac Newton trust, The Royal Society-Brian Mercer Award for Innovation and the European Research Council Grant NANOPOTS.

\section{Author information}

Reprints and permission information is available online at http://npg.nature.com/reprintsandpermissions/. Correspondence and requests for materials should be addressed to A.C.F. 